\title{
Long-range coherence between seismic noise properties in Japan and California before and after Tohoku mega-earthquake
}

\author{
A. A. Lyubushin ${ }^{1}$
}

Received: 29 February 2016/Accepted: 28 July 2016/Published online: 11 August 2016

(C) Akadémiai Kiadó 2016

\begin{abstract}
The coherences between daily time series of four low-frequency seismic noise properties which were calculated for 78 broadband seismic stations of the network F-net in Japan and 81 broadband seismic stations in California for 13 years of observation, 2003-2015, is investigated. The studied time interval includes Tohoku mega-earthquake, M9, on March 11, 2011. The chosen noise properties are the following: minimum normalized entropy of squared wavelet coefficients, multifractal singularity spectrum support width, generalized Hurst exponent and index of linear predictability. These properties were estimated daily as median values taken over all stations of the networks. For each pair of these noise properties from Japan and California squared coherence spectrums were estimated within moving time window of the length 730 days. The maximum values of squared coherence spectra for periods more than 20 days were essentially increasing as the time window approaches the time moment of Tohoku mega-earthquake and achieved their maximum values for position of moving time window strictly before the seismic catastrophe. This fact is interpreted as a consequence of general global seismic noise synchronization before huge seismic catastrophe.
\end{abstract}

Keywords Seismic noise $\cdot$ Multifractals $\cdot$ Entropy $\cdot$ Wavelets $\cdot$ Synchronization

\section{Introduction}

The low-frequency microseismic oscillations and their correlation with the processes occurring in the hydrosphere and atmosphere of the Earth, which are the major sources of microseismic energy, are a common subject of research in geophysics (Kobayashi and Nishida 1998; Tanimoto 2001, 2005; Rhie and Romanowicz 2004). It is however evident

\section{A. A. Lyubushin}

lyubushin@yandex.ru

1 Institute of the Physics of the Earth, Russian Academy of Sciences, Moscow, Russia 
that the variations in the structure of the microseismic background may also reflect the changes in the properties of the Earth's crust, which is the medium where the microseismic signals propagate.

Variations of the low-frequency noise properties from different parts of the Earth have a rather strong correlations and coherences. The seismic noise coherence effects were investigated in the papers (Lyubushin 2014a, 2015). It was shown that multiple spectral coherences and wavelet-based correlations are increasing in time and this increasing coincides with dramatic increasing of strongest earthquakes rate which is observed starting from Sumatra mega-earthquake at 26 Dec of 2004, especially starting from 2007. Now we try to investigate coherences between pairs of 4 integral (i.e. calculated by information from all stations of networks) seismic noise properties for two dense clusters of broadband seismic stations-network F-net in Japan and joint of three regional networks in California and try to answer on the question whether approaching to the seismic catastrophe is accompanied by increasing of coherence between seismic noise properties in these regions which are placed at such big distance from each other.

\section{Data}

For the analysis a vertical broadband seismic oscillations components with 1-s sampling time step (LHZ-records) from the broadband seismic networks in Japan and California were downloaded from the beginning of 2003 up to the end of 2015. This time interval has a length 13 years and includes time moments of Tohoku mega-earthquake in Japan at March 11, 2011.

Data from Japan were taken from 78 stations of the network F-net (Fig. 1a) from the address:http://www.fnet.bosai.go.jp/faq/?LANG=en.

Data from California were taken from 81 stations of three regional networks (Fig. 1b) which are presented by addresses: http://ds.iris.edu/mda/AZ, http://ds.iris.edu/mda/BK, http://ds.iris.edu/mda/CI.
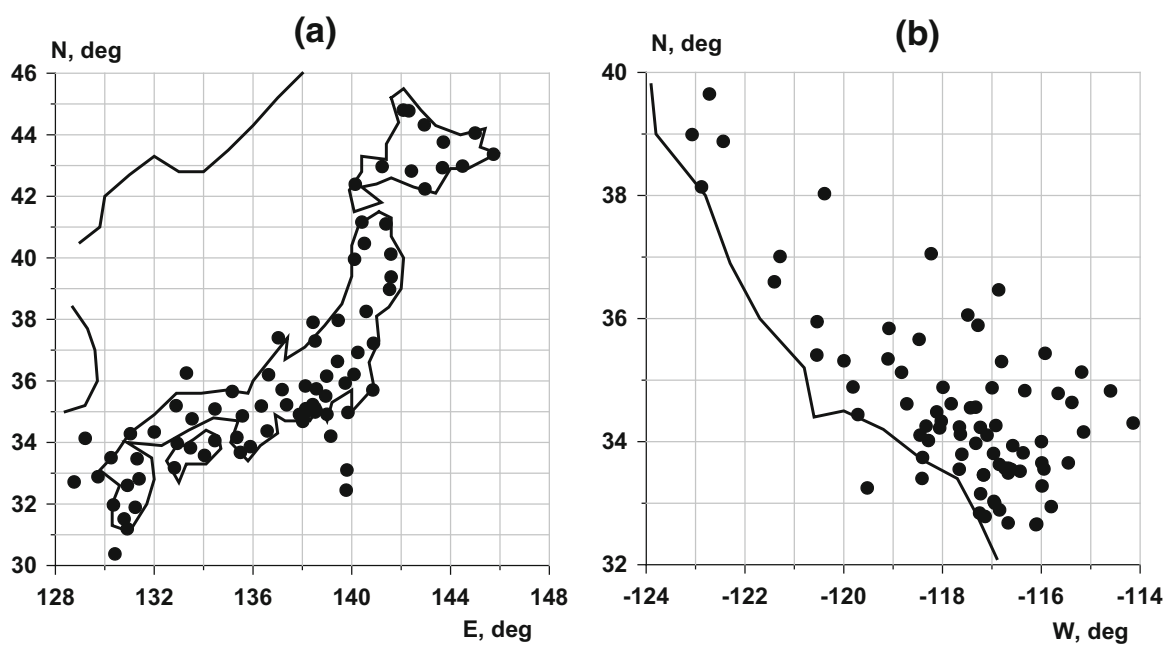

Fig. 1 Positions of broadband seismic stations in Japan (Fig. 1a-78 stations) and in California (Fig. 1b81 stations) 
Initial data are provided by seismic sensors STS-1 and STS-2, the units in records are given as velocity in meters per second and they were taken from these sites "as is". In this paper the seismic data were analysed after transforming them to sampling time step $1 \mathrm{~min}$ by calculating mean values within adjacent time windows of the length $60 \mathrm{~s}$. Thus, the minimum period of seismic noise variations for the analysis equals $2 \mathrm{~min}$. No other preliminary processing operations of the seismic records were performed. It should be noticed that four seismic noise properties which are analysed in this paper are dimensionless and are invariant to the scale of the records.

\section{Seismic noise statistics}

\subsection{Minimum normalized entropy $E n$ of squared wavelet coefficients}

Let $x(t)$ be the finite sample of the signal $t=1, \ldots, N$-index, numerating the counts. The normalized entropy is defined by the formula:

$$
E n=-\sum_{k=1}^{N} p_{k} \cdot \log \left(p_{k}\right) / \log (N), p_{k}=c_{k}^{2} / \sum_{j=1}^{N} c_{j}^{2}, \quad 0 \leq E n \leq 1
$$

Here $c_{k}, k=1, N$ are the orthogonal wavelet coefficients which were found from minimized the value (1). We try 17 orthogonal wavelets (Mallat 1998): 10 usual wavelets of Daubechies (number of vanishing moments equals to integer numbers from 1 up to 10) and 7 a so called symlets with numbers of vanishing moments varying from 4 up to 10. For low-frequency noise the parameters $E n$ were estimated within adjacent time windows of the length $N=1440$, i.e. 1 day, after removing trend by polynomial of the 8 -th order. Minimum normalized entropy En was suggested in (Lyubushin 2012) and was used for investigating sesimic noise properties in (Lyubushin 2013a, b, 2014a; Lyubushin et al. 2014).

\subsection{Multifractal parameters $\Delta \alpha$ and $\alpha^{*}$}

Multifractal singularity spectrum $F(\alpha)$ (Feder 1988) of the signal $x(t)$ is defined as a fractal dimensionality of time moments $t_{\alpha}$ which have the same value of local Lipschitz-Holder exponent: $h(t)=\lim _{\delta \rightarrow 0}(\ln (\mu(t, \delta)) / \ln (\delta))$, i.e. $h\left(t_{\alpha}\right)=\alpha$, where $\mu(t, \delta)=(\max x(s)-$ $\min x(s)$ ), maximum and minimum values are taken for argument $t-\delta / 2 \leq s \leq t+\delta / 2$, where $\delta$ is the length of time interval. The value $\mu(t, \delta)$ is a measure of signal variability in the vicinity of time moment $t$ (Feder 1988). If $X(t)$ is a usual self-similar monofractal signal (Taqqu 1988) with Hurst exponent value $0<H<1$, then $F(H)=1, F(\alpha)=0 \forall \alpha \neq$ $H$ but finite sample estimate of singularity spectrum does not obey these rigorous theoretical conditions of course.

Practically the most convenient method for estimating singularity spectrum is a method of multifractal detrended fluctuations analysis (DFA) (Kantelhardt et al. 2002) which is used here. The function $F(\alpha)$ could be characterized by following parameters: $\alpha_{\min }, \alpha_{\max }, \Delta \alpha=\alpha_{\max }-\alpha_{\min }$ and $\alpha^{*}$-an argument providing maximum to singularity spectra: $F\left(\alpha^{*}\right)=\max _{\alpha} F(\alpha)$. Parameter $\alpha^{*}$ is called a generalized Hurst exponent and it gives the most typical value of Lipschitz-Holder exponent. Parameter $\Delta \alpha$, singularity spectrum support width, could be regarded as a measure of variety of stochastic behavior. 
For removing scale-dependent trends (which are mostly caused by tidal variations) in multifractal DFA-method of singularity spectrums estimates a local polynomials of the 8-th order were used.

Multifractal analysis is a rather popular tool in geophysical studies (Ramirez-Rojas et al. 2004; Ida et al. 2005; Currenti et al. 2005; Telesca et al. 2005; Lyubushin et al. 2012). Estimates of multifractal properties $\Delta \alpha$ and $\alpha^{*}$ of low-frequency seismic noise were used in the papers Lyubushin (2008, 2010, 2011a, b, 2013a, b, 2014b, 2015) for the purposes of earthquake prediction and dynamic estimate of seismic danger.

\subsection{Index of linear predictability $c$ Pred}

This index was proposed in (Lyubushin 2010), see also (Lyubushin 2011b, 2012). Let $x(t)$ be the recorded signal. Let us take "long" adjacent time windows of the length $N$ counts and consider "short" time window of the length $n$ counts, $n<N$, which is moving from left to right direction within each "long" adjacent window with minimum mutual shift 1 sample. These "short" time windows are used for constructing two predictors one step ahead within each "long" window.

The 1-st predictor is trivial and for each time moment $t>n, t=1, \ldots, N$, within "long" window, it is calculated as mean value over previous "short" window: $\hat{x}_{0}(t)=$ $\sum_{s=t-n}^{t-1} x(s) / n$. Thus, we can compute the error of trivial predictor: $\varepsilon_{0}(t)=x(t)-\hat{x}_{0}(t)$ and its variance: $V_{0}=\sum_{t=n+1}^{N} \varepsilon_{0}^{2}(t) /(N-n)$.

The 2nd predictor is based on using correlations between neighbor values of the signal $x(t)$ and use the autoregression model $\mathrm{AR}(2)$ of 2-nd order: $\hat{x}_{A R}(t)=a_{1} x(t-1)+a_{2}$ $x(t-2)+d, \quad t>n$.

Vector of AR(2)-parameters $c=\left(a_{1}, a_{2}, d\right)^{T}$ is defined by least squares method using values of the signal $x(t)$ within "short" time window of the length $n$ which is adjacent to time moment $t>n$ from left-hand side:

$$
\hat{c}(t)=A^{-1}(t) \cdot R(t), A(t)=\sum_{s=t-(n-2)}^{t-1} Y(s) \cdot Y^{T}(s), \quad R(t)=\sum_{s=t-(n-2)}^{t-1} x(s) \cdot Y(s)
$$

where $Y(s)=(x(s), x(s-1), 1)^{T}$. Similar to trivial predictor we can compute the error of $\mathrm{AR}(2)$-predictor: $\varepsilon_{A R}(t)=x(t)-\hat{x}_{A R}(t)$ and its variance $V_{A R}=\sum_{t=n+1}^{N} \varepsilon_{A R}^{2}(t) /(N-n)$. Index of linear predictability is defined as $c$ Pred $=V_{0} / V_{A R}-1$.

The 2nd autoregression model $\mathrm{AR}(2)$ is selected because this is the minimal order for the AR-model, which enables one to describe the oscillatory motion and could provide the maximum spectral density between the Nyquist frequency and zero (Box and Jenkins 1970; Kashyap and Rao 1976). The AR-prediction makes use of the correlation property of the nearby values of increments of the recorded signals. If such a correlation exists and signal $x(t)$ is linearly predictable within current "long" time window of the length $N$ sample then $V_{A R}<V_{0}$, and $c$ Pred $>0$.

\section{Spectral measure of coherence}

Here we apply spectral measure of coherence $\lambda(\tau, \omega)$ which was suggested in (Lyubushin, $1998,1999)$ for multidimensional time series processing in the problems of geophysical monitoring. This spectral measure was applied for investigating synchronization effects in 
multidimensional time series in seismology (Lyubushin 2008, 2009, 2010, 2014a), hydrology (Lyubushin et al. 2004) and climate researches (Lyubushin and Klyashtorin 2012). Here we use it in the particular case of 2-dimensional time series (Lyubushin et al. 2015). In general case $\lambda(\tau, \omega)$ is constructed as the module of the product of componentby-component canonical coherences

$$
\lambda(\tau, \omega)=\prod_{j=1}^{m}\left|v_{j}(\tau, \omega)\right|
$$

Here, $m \geq 2$ is the total number of jointly analysed time series; $\omega$ is frequency; $\tau$ is the time coordinate of the right-hand end of the moving time window consisting of a definite number of adjacent samples; and $v_{j}(\tau, \omega)$ is the canonical coherence of the $j$-th scalar time series, which describes the strength of coupling of this series with all other series. The quantity $\left|v_{j}(\tau, \omega)\right|^{2}$ is the generalization of the ordinary squared spectrum of coherence between two signals for the case, when the second signal is not scalar but vector. The inequality $0 \leq\left|v_{j}(\tau, \omega)\right| \leq 1$ is fulfilled, and the closer the value of $\left|v_{j}(\tau, \omega)\right|$ to unity, the stronger the linear relation of variations at the frequency $\omega$ in the time window with the coordinate $\tau$ of the $j$-th series to analogous variations in all other series.

For calculating the measure (3) it is necessary to estimate spectral matrix $S(\tau, \omega)$ of the size $m \times m$ within each time window with time coordinate $\tau$. For this purpose we use vector autoregression model (Marple 1987):

$$
Z(t \mid \tau)+\sum_{k=1}^{p} A_{k}(\tau) \cdot Z(t-k \mid \tau)=e(t \mid \tau)
$$

where $t$ is time index within current time window with time coordinate $\tau, Z(t \mid \tau)$ is the piece of $m$-dimensional time series corresponding to the current time window, $p$ is an autroregression order, $A_{k}(\tau)$ are matrices of autoregression coefficients of the size $m \times m, e(t \mid \tau)$ is $m$-dimensional residual signal with zero mean and covariance matrix $\Phi(\tau)=M\{e(t \mid \tau)$ $\left.e^{T}(t \mid \tau)\right\}$. Matrices $A_{k}(\tau)$ and $\Phi(\tau)$ are defined in each time window using DurbinLevinson procedure and the spectral matrix is calculated using formula:

$$
S(\tau, \omega)=F^{-1}(\tau, \omega) \cdot \Phi(\tau) \cdot F^{-H}(\tau, \omega), \quad F(\tau, \omega)=E+\sum_{k=1}^{p} A_{k}(\tau) \cdot \exp (-i \omega k)
$$

where $E$ is a unit matrix of the size $m \times m$, " $H$ " is the sign of Hermitian conjunctions.

\section{Results}

For low-frequency noise the linear predictability index $c$ Pred was estimated for increments of waveforms. The transition to increments is dictated by the necessity to avoid dominance of low frequencies associated with tides and other trends. In the calculations of the linear predictability index for 1-min data, the estimations were performed in the adjacent long time windows of length $N=1440$, i.e. 1 day. The "short" time window length $n=60$, i.e. $1 \mathrm{~h}$.

After estimating daily values of statistics $E n, \Delta \alpha, \alpha^{*}$ and $c$ Pred from each station of the network we can calculate their median values and thus construct 4 daily time series which characterize integral properties of each network. Figure 2 presents graphics of these daily 

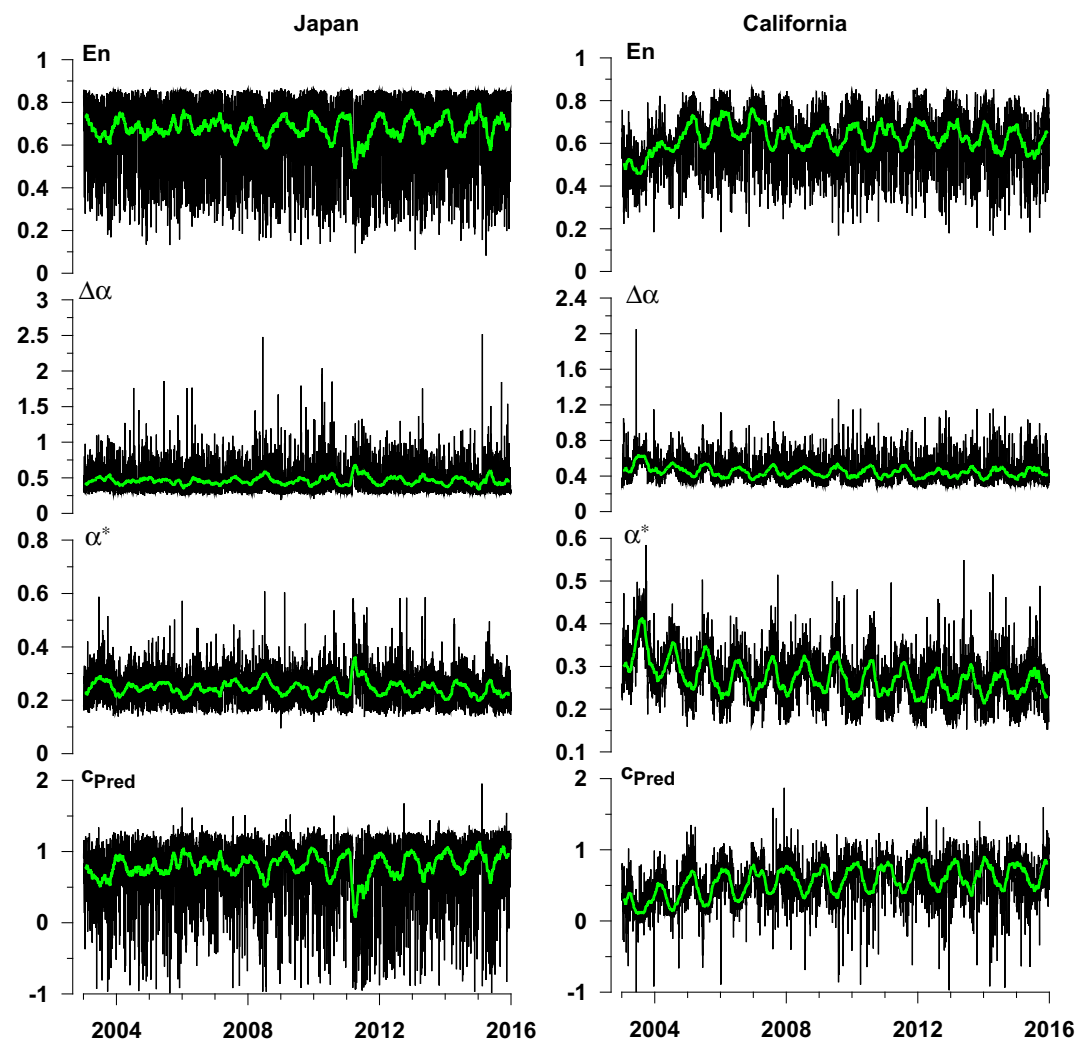

Fig. 2 Graphics of median values of 4 daily seismic noise properties calculated over all stations in Japan (left-hand column of graphics, stations from Fig. 1a) and in California (right-hand column of graphics, stations from Fig. 1b): En-minimum normalized entropy of squared wavelet-coefficients; $\Delta \alpha-$ multifractal singularity spectrum support width; $\alpha^{*}$-generalized Hurst exponent; $c$ Pred-index of linear predictability. Bold green lines present running average within moving time window of the length 57 days

time series (4383 samples within each series) noise properties in Japan and California. The annual variations of the seismic noise properties could easily be notices, especially from the behaviour of running average curves (bold green lines). We suppose that annual variations are caused by changing in the structure of seismic noise as the result of influence of winter storms in the ocean (Rhie and Romanowicz 2004; Tanimoto 2005).

The next step of investigation is estimate of evolution of squared coherence spectra for each of four pairs of time series presented at Fig. 2, from its left and right columns. We applied the spectral measure (3) for the case when $m=2$ within time window of the length 730 daily samples with mutual shift seven samples. We used autoregression order $p=5$ in the formula (4). The choice of the 2-years length of moving time window is following from the purpose to get rid of the influence of seasonal 1-year variations of seismic noise properties which are clearly seen at the Fig. 2.

In general case when $m>2$ the value (3) is calculated using canonical coherences of the $m$-dimensional time series $Z(t)$ (Lyubushin 1998, 1999), but for our particular case when $m=2$ the value (3) equals to $\left|S_{12}(\tau, \omega)\right|^{2} /\left(S_{11}(\tau, \omega) \cdot S_{22}(\tau, \omega)\right)$, where $S_{11}(\tau, \omega)$ and $S_{22}(\tau, \omega)$ are diagonal elements of the matrix (5), i.e. parametric estimates of the power 
spectra of two signals within time window with time coordinate $\tau$, and $S_{12}(\tau, \omega)$ is their mutual cross-spectrum.

Time-frequency diagrams of squared coherence spectrums are presented at the Figs. 3 , 4,5 and 6 . It could be noticed that most of maximum values of squared coherence belong to the frequency range corresponding to periods more than 20 days and that peak values are achieved for position of moving time window which is close to time moment of Tohoku mega-earthquake (time mark 2011.189) and laying strictly before this time moment. At the beneath one-dimensional graphs at the Figs. 3, 4, 5 and 6 these evolutions are presented for squared coherence spectra maximums which are calculated within each time window for frequency values corresponding to periods more than 20 days. From the Figs. 3, 4, 5 and 6 we see that time moments before earthquake is characterized by

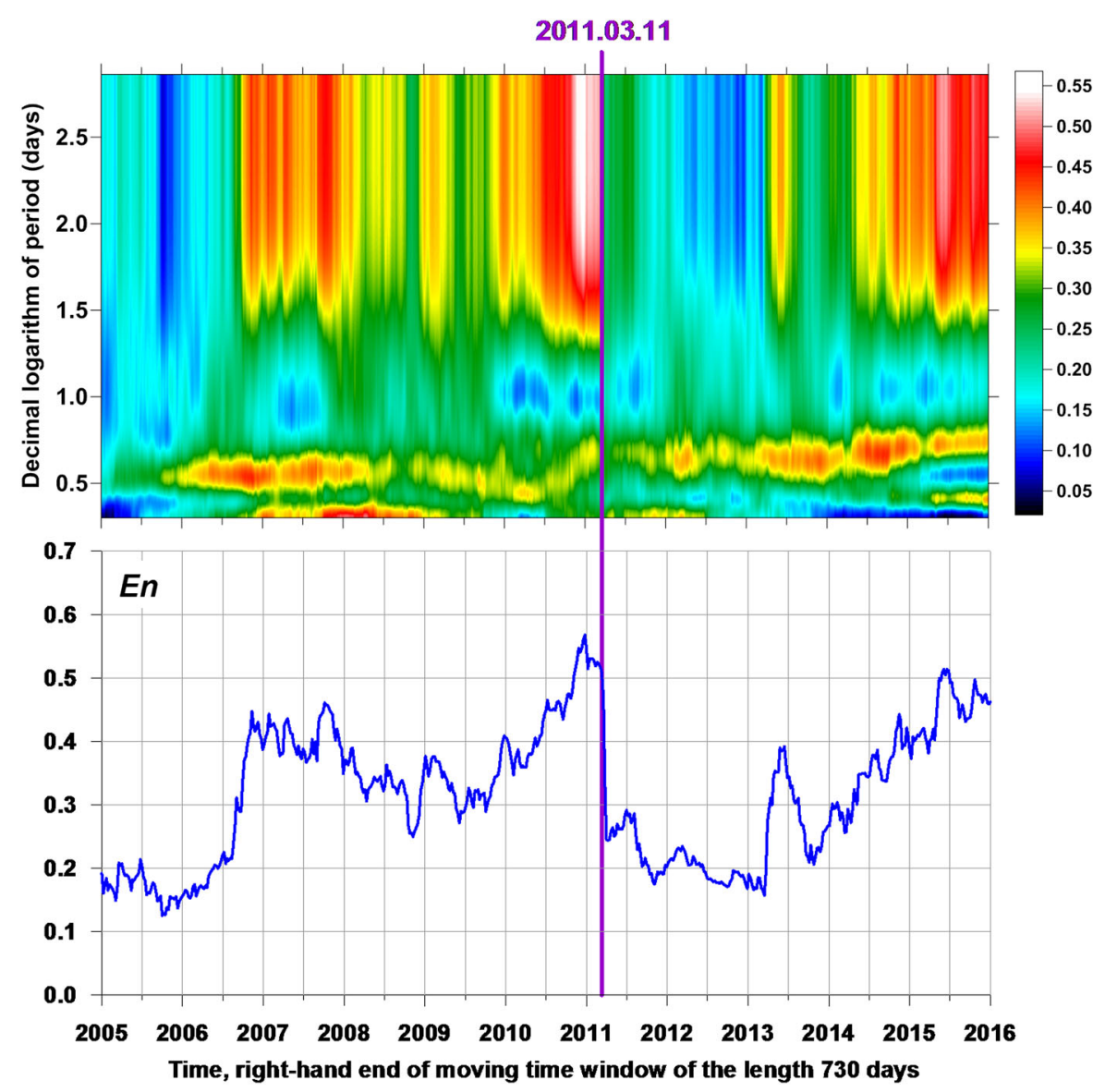

Fig. 3 Time-frequency diagram of evolution of squared coherence spectrum between median values of minimum normalized entropy $E n$ of squared wavelet-coefficients for daily seismic noise waveforms from broadband seismic networks in Japan and California in dependence on right-hand end position of moving time window of the length 730 days taken with mutual shift 7 days. The beneath graph presents maximum values of squared coherence spectrum in each window with respect to frequency values corresponding to periods more than 20 days. The bold purple vertical line indicates time moment of Tohoku mega-earthquake in Japan on 11 March, 2011, M = 9 


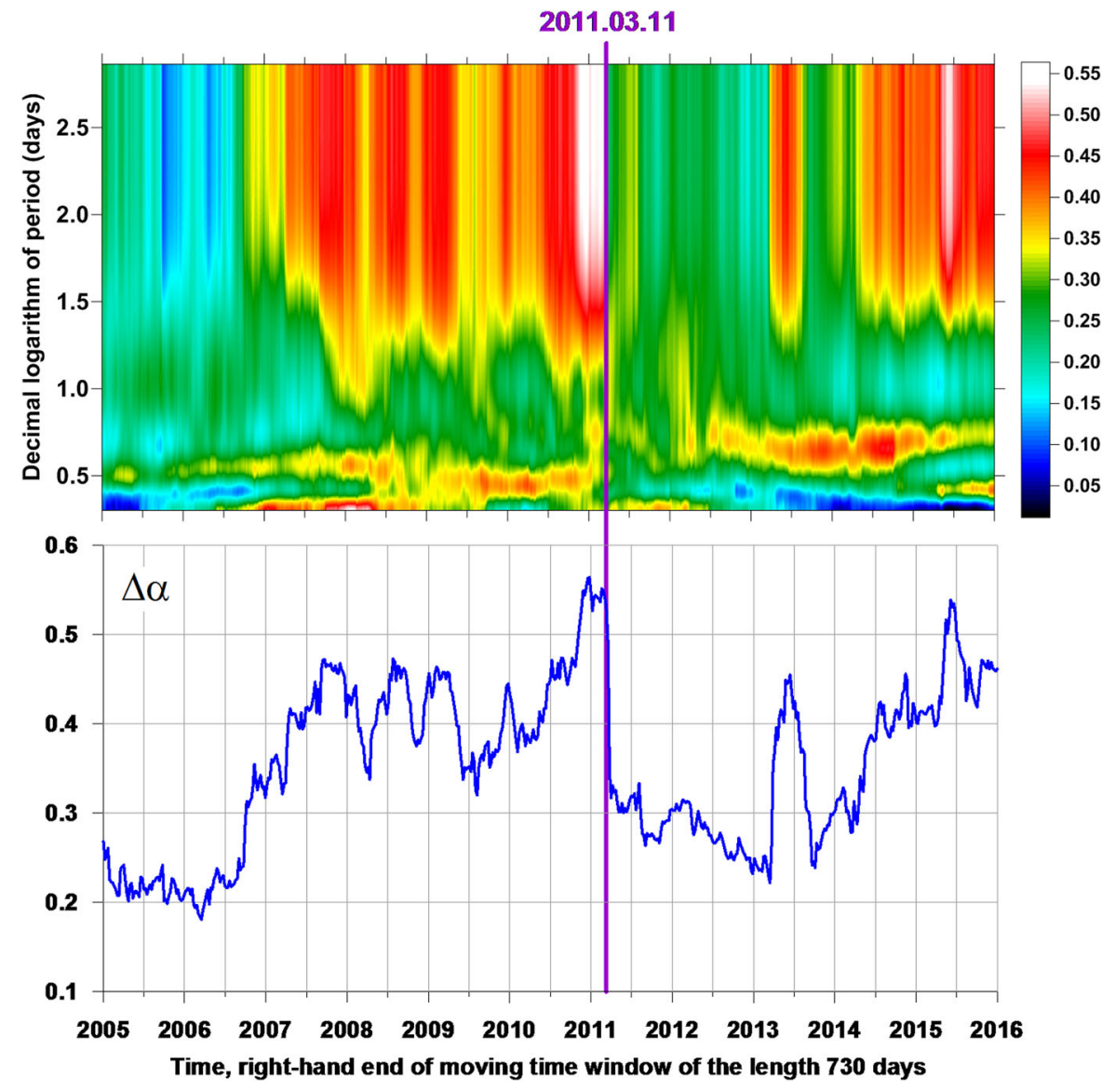

Fig. 4 Time-frequency diagram of evolution of squared coherence spectrum between median values of multifractal singularity spectrum support width $\Delta \alpha$ for daily seismic noise waveforms from broadband seismic networks in Japan and California in dependence on right-hand end position of moving time window of the length 730 days taken with mutual shift 7 days. The beneath graph presents maximum values of squared coherence spectrum in each window with respect to frequency values corresponding to periods more than 20 days. The bold purple vertical line indicates time moment of Tohoku mega-earthquake in Japan on 11 March, 2011, $\mathrm{M}=9$

increasing trend of coherence behaviour and that maximum values correspond to position of time window directly before the seismic event.

\section{Conclusion}

Strong effect of increasing coherence between behaviour of different parameters of lowfrequency seismic noise in Japan and California before Tohoku mega-earthquake on March 11, 2011 in Japan is detected by analysis of seismic noise waveforms from broadband seismic networks.

Results presented at the Figs. 3, 4, 5 and 6 show that there is an explicit precursory increasing of coherence in the behaviour of seismic noise properties in Japan and 


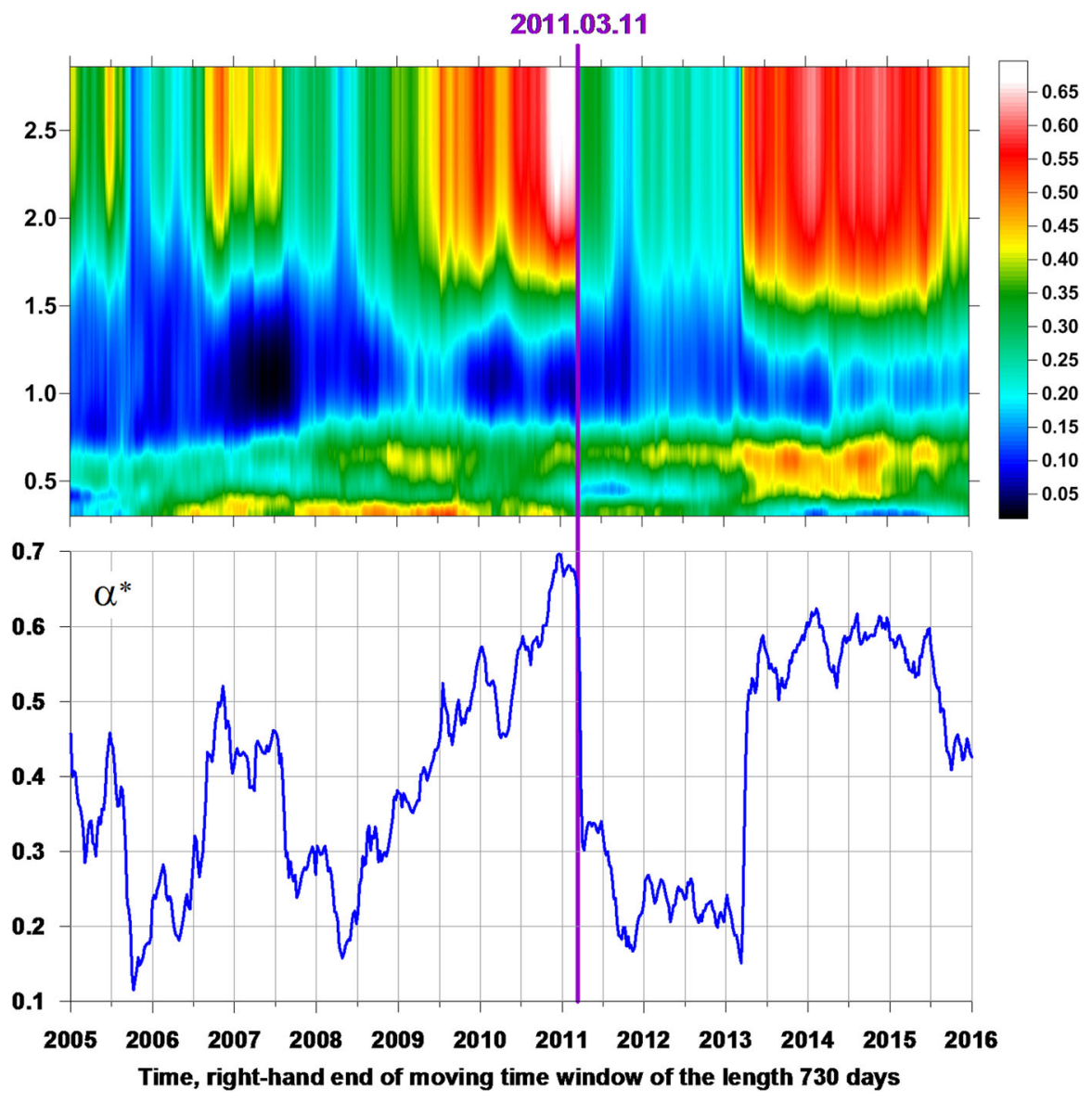

Fig. 5 Time-frequency diagram of evolution of squared coherence spectrum between median values of multifractal generalized Hurst exponent $\alpha^{*}$ for daily seismic noise waveforms from broadband seismic networks in Japan and California in dependence on right-hand end position of moving time window of the length 730 days taken with mutual shift 7 days. The beneath graph presents maximum values of squared coherence spectrum in each window with respect to frequency values corresponding to periods more than 20 days. The bold purple vertical line indicates time moment of Tohoku mega-earthquake in Japan on 11 March, 2011, M = 9

California before the Tohoku mega-earthquake despite the big distance between seismic networks. This could be explained by general phenomenon of increasing of correlation radius of noise fluctuations within complex system (Earth's crust in our case) when the system is approaching to abrupt change of its properties (Gilmore 1981; Nicolis and Prigogine 1989).

I understand that any speculations and hypotheses about the possibility of earthquake prediction and the existence of earthquake precursors currently are suspicious and go beyond the generally accepted rules in scientific articles. That is why I brought only the facts about the experimental discovery of long-term positive trend in the coherence between the properties of seismic noise in such remote regions as Japan and California. It should be emphasized that the growth of coherence was detected for four seismic noise 


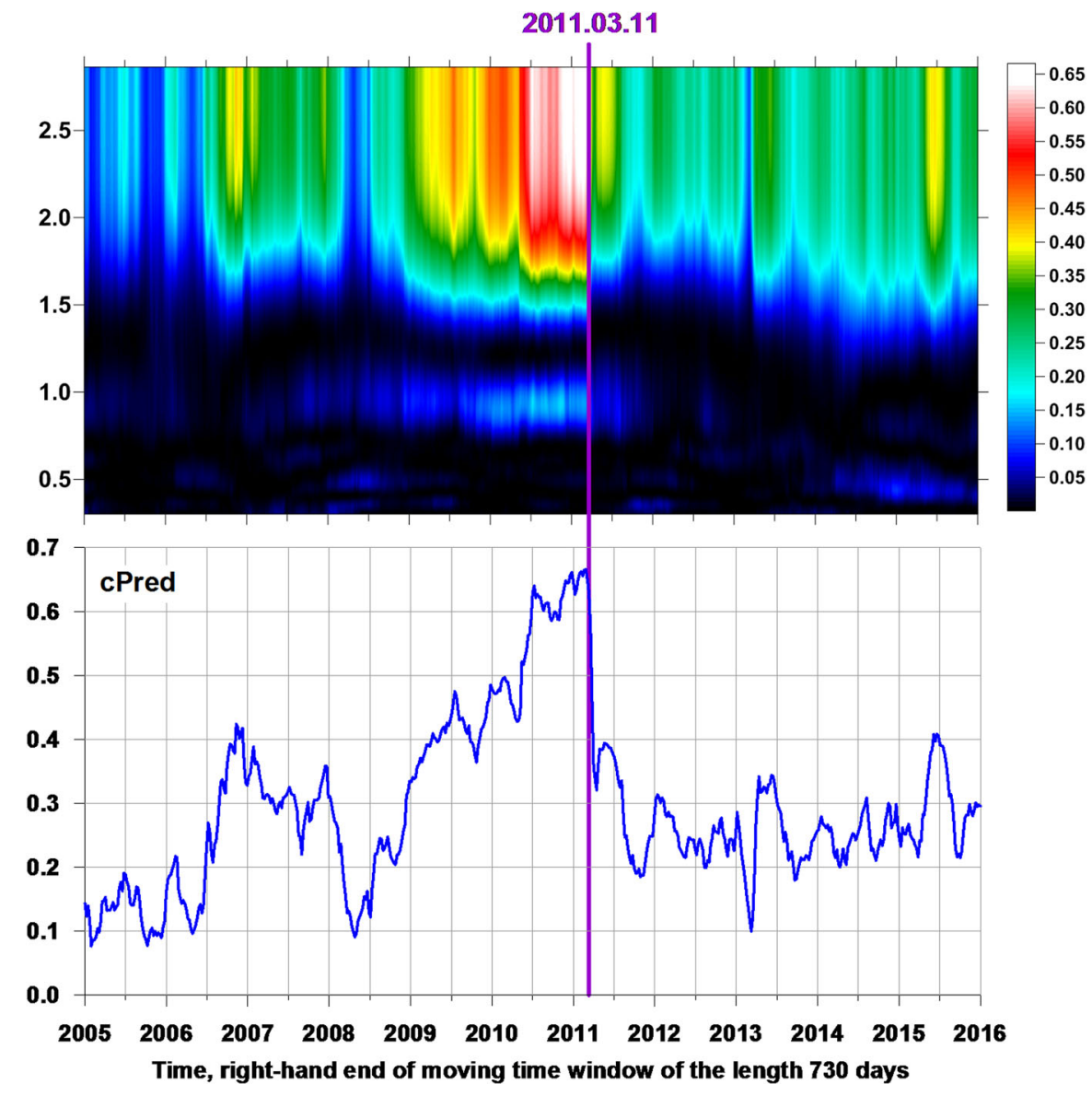

Fig. 6 Time-frequency diagram of evolution of squared coherence spectrum between median values of linear predictability index $c$ Pred for daily seismic noise waveforms from broadband seismic networks in Japan and California in dependence on right-hand end position of moving time window of the length 730 days taken with mutual shift 7 days. The beneath graph presents maximum values of squared coherence spectrum in each window with respect to frequency values corresponding to periods more than 20 days. The bold purple vertical line indicates time moment of Tohoku mega-earthquake in Japan on 11 March, 2011, $\mathrm{M}=9$

properties that are significantly different from each other in their meaning and calculation methods. Since the main conclusions of this paper were obtained by joint processing of long seismic records and subsequent deep averaging of the results of the analysis by a large number of stations, the probability of random coherence growth is negligible. Any artificial factors, e.g. time variation of mechanical/electrical property change of seismographs could scarcely be the reason of coherence growth as well because such kind of changes could not be synchronized in Japan and California simultaneously.

The effects of increasing of seismic noise coherence before Tohoku mega-earthquake and before and after Sumatra mega-earthquake on 26 Dec, 2004, M = 9.1-9.3, were previously investigated in the papers (Lyubushin 2009, 2010, 2014a, 2015). 
Acknowledgments The work was financially supported by Russian Foundation for Basic Research (Project No. 15-05-00414) and the Ministry of Education and Science of the Russian Federation (in accordance with the requirements of the Contract No. 14.577.21.0109)

\section{References}

Box GEP, Jenkins GM (1970) Time series analysis. Forecasting and control. Holden-Day, San Francisco Currenti G, del Negro C, Lapenna V, Telesca L (2005) Multifractality in local geomagnetic field at Etna volcano, Sicily (southern Italy). Nat Hazards Earth Syst Sci 5:555-559. doi:10.5194/nhess-5-555-2005

Feder J (1988) Fractals. Plenum Press, New York

Gilmore R (1981) Catastrophe theory for scientists and engineers. Wiley, New York

Ida Y, Hayakawa M, Adalev A, Gotoh K (2005) Multifractal analysis for the ULF geomagnetic data during the 1993 Guam earthquake. Nonlinear Process Geophys 12:157-162. doi:10.5194/npg-12-157-2005

Kantelhardt JW, Zschiegner SA, Konscienly-Bunde E, Havlin S, Bunde A, Stanley HE (2002) Multifractal detrended fluctuation analysis of nonstationary time series. Phys A 316:87-114. doi:10.1016/s03784371(02)01383-3

Kashyap RL, Rao AR (1976) Dynamic stochastic models from empirical data. Academic Press, New York

Kobayashi N, Nishida K (1998) Continuous excitation of planetary free oscillations by atmospheric disturbances. Nature 395:357-360. doi:10.1038/26427

Lyubushin AA (1998) Analysis of canonical coherences in the problems of geophysical monitoring. Izv Phys Solid Earth 34(1):52-58

Lyubushin AA (1999) Analysis of multidimensional geophysical monitoring time series for earthquake prediction. Ann Geofis 42(5):927-937. doi:10.4401/ag-3757

Lyubushin AA (2008) Microseismic noise in the low frequency range (periods of 1-300 min): properties and possible prognostic features. Izv Phys Solid Earth 44(4):275-290. doi:10.1134/S106935130 8040022

Lyubushin AA (2009) Synchronization trends and rhythms of multifractal parameters of the field of lowfrequency microseisms. Izv Phys Solid Earth 45(5):381-394. doi:10.1134/S1069351309050024

Lyubushin A (2010) Multifractal parameters of low-frequency microseisms. In: de Rubeis V et al. (ed) Synchronization and triggering: from fracture to earthquake processes. GeoPlanet: Earth Planet Sci 1, doi:10.1007/978-3-642-12300-9_15. Springer, Berlin 2010, Chapter 15, pp 253-272

Lyubushin AA (2011a) Cluster analysis of low-frequency microseismic noise. Izv Phys Solid Earth 47(6):488-495. doi:10.1134/S1069351311040057

Lyubushin AA (2011b) Seismic catastrophe in Japan on March 11, 2011: long-term prediction on the basis of low-frequency microseisms. Izv Atmos Ocean Phys 46(8):904-921. doi:10.1134/S00014338 11080056

Lyubushin A (2012) Prognostic properties of low-frequency seismic noise. Nat Sci 4:659-666. doi:10.4236/ ns.2012.428087

Lyubushin AA (2013a) Mapping the properties of low-frequency microseisms for seismic hazard assessment. Izv Phys Solid Earth 49(1):9-18. doi:10.1134/S1069351313010084

Lyubushin A (2013b) How soon would the next mega-earthquake occur in Japan? Nat Sci 5(8A1):1-7. doi:10.4236/ns.2013.58A1001

Lyubushin AA (2014a) Analysis of coherence in global seismic noise for 1997-2012. Izv Phys Solid Earth 50(3):325-333. doi:10.1134/S1069351314030069

Lyubushin AA (2014b) Dynamic estimate of seismic danger based on multifractal properties of lowfrequency seismic noise. Nat Hazards 70(1):471-483. doi:10.1007/s11069-013-0823-7

Lyubushin AA (2015) Wavelet-based coherence measures of global seismic noise properties. J Seismol 19(2):329-340. doi:10.1007/s10950-014-9468-6

Lyubushin AA, Klyashtorin LB (2012) Short term global $d T$ prediction using (60-70)-years periodicity. Energy Environ 23(1):75-85. doi:10.1260/0958-305X.23.1.75

Lyubushin AA, Pisarenko VF, Bolgov MV, Rodkin MV, Rukavishnikova TA (2004) Synchronous variations in the Caspian Sea level from coastal observations in 1977-1991. Atmos Ocean Phys 40(6):737-746

Lyubushin AA, Kaláb Z, Lednická M (2012) Geomechanical time series and its singularity spectrum analysis. Acta Geod Geophys Hung 47(1):69-77. doi:10.1556/AGeod.47.2012.1.6

Lyubushin AA, Kaláb Z, Lednická M (2014) Statistical properties of seismic noise measured in underground spaces during seismic swarm. Acta Geod et Geophys 49(2):209-224. doi:10.1007/s40328-014-0051-y 
Lyubushin AA, Kaláb Z, Lednická M, Knejzlik J (2015) Coherence spectra of rotational and translational components of mining induced seismic events. Acta Geod et Geophys. doi:10.1007/s40328-015-0099-3

Mallat S (1998) A wavelet tour of signal processing. Academic, San Diego, p 577

Marple SL Jr (1987) Digital spectral analysis with applications. Prentice-Hall Inc, Englewood Cliffs

Nicolis G, Prigogine I (1989) Exploring complexity, an introduction. W.H. Freedman and Co., New York

Ramirez-Rojas A, Munoz-Diosdado A, Pavia-Miller CG, Angulo-Brown F (2004) Spectral and multifractal study of electroseismic time series associated to the $\mathrm{Mw}=6.5$ earthquake of 24 October 1993 in Mexico. Nat Hazards Earth Syst Sci 4:703-709

Rhie J, Romanowicz B (2004) Excitation of Earth's continuous free oscillations by atmosphere-oceanseafloor coupling. Nature 431:552-554. doi:10.1038/nature02942

Tanimoto T (2001) Continuous free oscillations: atmosphere-solid earth coupling. Annu Rev Earth Planet Sci 29:563-584

Tanimoto T (2005) The oceanic excitation hypothesis for the continuous oscillations of the Earth. Geophys J Int 160:276-288. doi:10.1111/j.1365-246X.2004.02484.x

Taqqu MS (1988) Self-similar processes. Encyclopedia of statistical sciences, vol 8. Wiley, New York, pp 352-357

Telesca L, Colangelo G, Lapenna V (2005) Multifractal variability in geoelectrical signals and correlations with seismicity: a study case in southern Italy. Nat Hazards Earth Syst Sci 5:673-677 